\title{
Necessity and Challenges of Sample Preconcentration in Analysis of Multiple MicroRNAs by Capillary Electrophoresis
}

\author{
Liang Hu, ${ }^{1}$ Svetlana M. Krylova, ${ }^{1}$ Stanley K. Liu, ${ }^{2}$ George M. Yousef, ${ }^{3}$ and Sergey N. Krylov* \\ ${ }^{1}$ Department of Chemistry and Centre for Research on Biomolecular Interactions, York University, Toronto, Ontario M3J 1P3, Canada \\ ${ }^{2}$ Department of Radiation Oncology, Sunnybrook-Odette Cancer Centre, 2075 Bayview Avenue, Toronto, Ontario M4N 3M5, Canada \\ ${ }^{3}$ Department of Pediatric Laboratory Medicine, Hospital for Sick Children, 555 University Avenue, Toronto, Ontario M5G 1X8, Canada \\ *Corresponding author's email: skrylov@yorku.ca
}

\begin{abstract}
Thousands of putative miRNA-based cancer biomarkers have been reported but none has been validated for approval by the Food and Drug Administration. One of the reasons for this alarming discrepancy is the lack of a method which is sufficiently robust for carrying out validation studies, which may require analysis of samples from hundreds of patients across multiple institutions and pooling the results together. Capillary electrophoresis (CE)-based hybridization assay proved to be more robust than reversed transcription polymerase chain reaction (the current standard) but its limit of quantification (LOQ) exceeds 10 pM while miRNA concentrations in cell lysates are below $1 \mathrm{pM}$. Thus, CE-based separation must be preceded by on-column sample preconcentration. Here we explain challenges of sample preconcentration for CE-based miRNA analyses and introduce a preconcentration method that can suit CE-based miRNA analysis utilizing peptide nucleic acid (PNA) hybridization probes. The method combines field-amplified sample stacking (FASS) with isotachophoresis (ITP). We proved that FASS-ITP could retain and concentrate both near-neutral PNA with highly-negatively charged PNA-miRNA hybrids. We demonstrated that preconcentration by FASS-ITP could be combined with the CE-based separation of the unreacted PNA probes from the PNA-miRNA hybrids and facilitate improvement in LOQ by a factor of 140, down to 0.1 pM. Finally, we applied FASS-ITP-CE for simultaneous detection of two miRNAs in crude cell lysates and proved that the method was robust when used in complex biological matrices. The 140-fold improvement in LOQ and the robustness to biological matrices will significantly expand the applicability of CE-based miRNA analysis, bringing it closer to becoming a practical tool for validation of miRNA biomarkers.
\end{abstract}

MicroRNAs (miRNAs) are short single-stranded RNA molecules (18-25 nucleotides) that function as posttranscriptional regulators in gene expression. ${ }^{1}$ The abnormal expression of small subsets of miRNA (typically fewer than 10) termed miRNA fingerprints have been found in tumors, suggesting that miRNA fingerprints could serve as cancer biomarkers. $^{2}$ The discovery of putative miRNA fingerprints can be performed with well established semi-quantitative methods capable of analyzing hundreds and thousands of miRNAs simultaneously, such as microarrays. ${ }^{3}$ Fewer than 100 patients are used typically for such fingerprint-discovery studies. ${ }^{4,5}$ The discovery stage is, thus, relatively simple, and, accordingly, thousands of putative miRNA fingerprints have been published to date. ${ }^{6}$ Validation of such putative miRNA fingerprints, however, requires a larger pool of patients (as many as a thousand) ${ }^{7}$ and a rugged quantitative method for simultaneous analysis of a few miRNAs comprising the fingerprint. Method ruggedness is necessary because the required for validation number of patients is rarely available in a single cancer centre and, thus, validation requires pooling together data across multiple institutions.

Reversed Transcription Polymerase Chain Reaction (RT-PCR) is a current standard method for miRNA-fingerprint validation, but RT-PCR has multiple issues that deem this method rather non-rugged. ${ }^{8}$ The lack of a rugged quantitative method for analysis of multiple miRNAs contributes to the fact that none of the thousands of putative miRNA fingerprints has been approved by the Food and Drug Administration to guide cancer therapy. A very similar situation with the difficulties of validation and approval can be found for other types of cancer biomarkers. ${ }^{9}$

There is an ongoing effort to find an alternative to RT-PCR for validation of miRNA fingerprints. ${ }^{10-16}$ A promising approach is direct quantitative analysis of multiple miRNAs (DQAMmiR) by capillary electrophoresis with laser-induced fluorescence detection (CE-LIF). ${ }^{17,18}$ DQAMmiR is a hybridization assay which utilizes fluorescently-labeled hybridization probes complementary to target miRNAs (Figure 1). The miRNA-probe hybrids are separated from each other and from the unreacted probes by $\mathrm{CE}$ and both the hybrids and the probes are detected with LIF. There are two implementations of DQAMmiR which differ by the nature of hybridization probes: ssDNA in the

\section{A. DQAMmiR utilizing ssDNA hybridization probes}

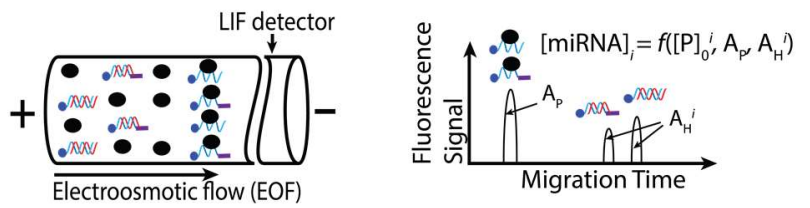

B. DQAMmiR utilizing PNA hybridization probes
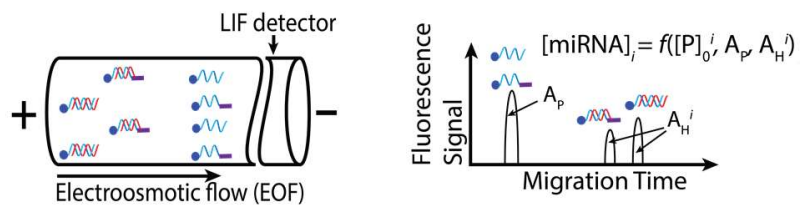

? 2 Fluorescently labeled hybridization probes

- men Probe-miRNA hybrids

ssDNA-binding protein (SSB)

Figure 1. Schematics of $C E$ separation in two implementations of DQAMmiR. ${ }^{17,18}$ In both cases, the concentrations of the miRNA targets are determined by analyzing peak areas in the electropherogram with a simple mathematical approach that requires knowing the total concentration of each probe, $[\mathrm{P}]_{0}{ }^{i}$, and a sum peak area of all the unreacted probes, $A_{\mathrm{P}}$, as well as a peak area of every hybrid, $A_{\mathrm{H}}{ }^{i}$. 
A

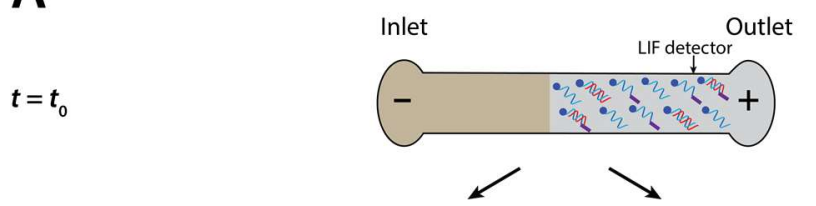

a. When using ssDNA hybridization probes

$\left|\mu_{\mathrm{TE}}\right|<\left|\mu_{\text {sSDAA }}\right| \approx\left|\mu_{\text {hybrid }}\right|<\left|\mu_{\mathrm{LE}}\right|$

Both the ssDNA-miRNA hybrids and the ssDNA probes focused by ITP

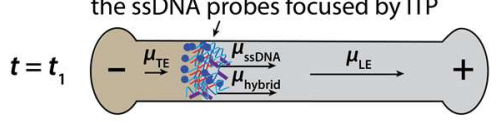

DQAMmiR succeeds after the CE separation resumes

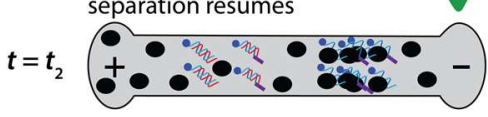

23 Hybridization probes

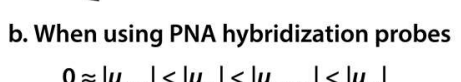

$$
0 \approx\left|\mu_{\mathrm{PNA}}\right|<\left|\mu_{\mathrm{TE}}\right|<\left|\mu_{\text {hybrid }}\right|<\left|\mu_{\mathrm{LE}}\right|
$$

The PNA-miRNA hybrids focused by ITP; the PNA probes eluted out of the capillary

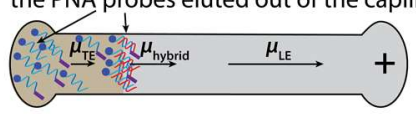

DQAMmiR fails because the unreacted PNA probes are lost

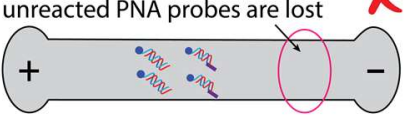

B

Hybridization mixture prepared in an extremely diluted buffer

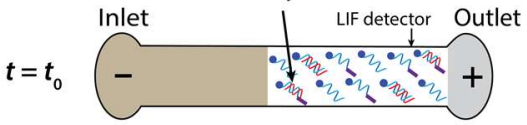

Both PNA probes and PNA-miRNA hybrids stacked into a narrow zone by FASS

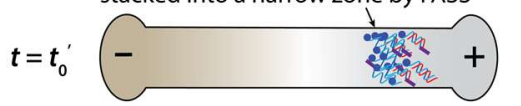

Hybrids focused at the TE/LE boundary by ITP; PNA probes still kept inside the capillary

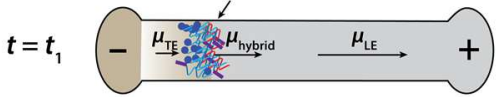

DQAMmiR succeeds after the $C E$ separation resumes

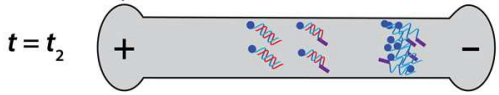

Figure 2. A) Conceptual depiction of ITP-CE for DQAMmiR with two different types of hybridization probes: ssDNA (a) and PNA (b). In both cases, a large plug of the hybridization mixture is prepared in the leading electrolyte (LE) and injected into the capillary, which is prefilled with the trailing electrolyte (TE), from the outlet vial. The outlet vial is then replaced with LE and a voltage is applied with the positive polarity being at the outlet $\left(t=t_{0}\right)$. In the case of ssDNA hybridization probes (a), because the electrophoretic mobilities of the ssDNA probes, $\mu_{\mathrm{ssDNA}}$, and the probe-miRNA hybrids, $\mu_{\mathrm{hybrid}}$, are less than that of the LE ion, $\mu_{\mathrm{LE}}$, and greater than that of the TE ion, $\mu_{\mathrm{TE}},\left(\left|\mu_{\mathrm{TE}}\right|<\left|\mu_{\mathrm{sSDA}}\right| \approx\left|\mu_{\mathrm{hybrid}}\right|<\left|\mu_{\mathrm{LE}}\right|\right)$, both the hybrids and the ssDNA probes are focused at the TE/LE interface by ITP $\left(t=t_{1}\right)$. The strong EOF from the anode to the cathode propels the focused analytes to near the inlet. The CE separation resumes after reversing the polarity and replacing the inlet vial with the SSB-supplemented LE buffer, which facilitates DQAMmiR $\left(t=t_{2}\right)$. However, while using PNA as hybridization probes (b), only the PNA-miRNA hybrids can be focused by ITP because the electrophoretic mobilities of PNA probes, $\mu_{\mathrm{PNA}}$, are too low $\left(0 \approx\left|\mu_{\mathrm{PNA}}\right|<\left|\mu_{\mathrm{TE}}\right|\right)$. Consequently, unreacted PNA probes in the hybridization mixture elute from the capillary during ITP $\left(t=t_{1}\right)$, which leads to their loss and failure of DQAMmiR $\left(t=t_{2}\right)$. B) Conceptual depiction of the proposed FASS-ITP-CE approach for DQAMmiR utilizing PNA probes. The hybridization mixture is prepared in a diluted buffer which has conductivity much lower than those of LE and TE. Because of the conductivity gradient between the sample plug and LE, both the PNA probes and the PNA-miRNA hybrids are stacked into a narrow zone by FASS regardless of the magnitudes of their electrophoretic mobilities $\left(t=t_{0}^{\prime}\right)$. Due to the co-presence of TE and LE in the capillary, the PNA-miRNA hybrids in the narrow zone can further be focused into a narrower band at the TE/LE interface by ITP, while the stacked PNA probes do not focus further but migrate along with the focused hybrids, keeping them inside the capillary during the step of hybrid concentration by ITP $\left(t=t_{1}\right)$. Once the FASS-concentrated probes and FASS-ITP-concentrated hybrids reach near the inlet end of the capillary, the polarity is reversed and the CE separation resumes facilitating $\operatorname{DQAMmiR}\left(t=t_{2}\right)$.

original implementation, which requires single-strand DNA binding protein (SSB) in the CE running buffer (Figure 1, top), ${ }^{17}$ and peptide nucleic acid (PNA) in the later implementation, which does not require SSB (Figure 1, bottom). ${ }^{18}$

Unlike RT-PCR, DQAMmiR does not involve enzymatic reactions (which are error-prone); therefore, DQAMmiR is more robust than RT-PCR. ${ }^{19}$ However, its robustness comes at an expense of relatively high concentration limit of quantitation (LOQ). A typical cell-lysate sample contains miRNAs at sub-pM concentrations (see Note S1), while the LOQ of commercial CELIF instruments is in the low pM range at best. ${ }^{18}$ Thus, highlyefficient and highly-reproducible preconcentration of the hybrids inside the capillary before CE separation is necessary to address the LOQ issue while maintaining assay robustness.

A number of on-column sample preconcentration (OSP) methods have been developed for improving LOQ of $\mathrm{CE}$ including field-amplified sample stacking (FASS), ${ }^{20,21}$ dynamic $\mathrm{pH}$ junction, ${ }^{22}$ sweeping, ${ }^{23}$ and isotachophoresis (ITP). ${ }^{24}$ All OSP methods use heterogeneous buffer systems to change the analyte's velocity at interfaces of two adjacent buffer zones. The velocity change, then, can facilitate analyte's focusing from a large sample plug into a narrow zone, providing up to 10,000 -fold improvement in LOQ. ${ }^{25}$ However, the OSP methods require suppression of electroosmotic flow (EOF) in the capillary for the best result as the mismatch of the local EOF velocities in the heterogeneous zones causes dispersion of the focused analyte zone. $^{26}$ The dispersion, in turn, results in very modest concentration factors, typically less than 10 , and poor analyte resolutions in the subsequent $\mathrm{CE}$ separation. ${ }^{20}$ This drawback limits the applicability of OSP methods to DQAMmiR because DQAMmiR relies on strong EOF in the capillary for separation of the unreacted probes from the negatively charged miRNA-probe hybrids.

The least EOF-sensitive OSP method is arguably ITP which can concentrate analytes between the leading electrolyte (LE) and the trailing electrolyte (TE) while the electrophoretic mobilities of the analytes are less than that of the LE ion and greater than that of the TE ion. ${ }^{24}$ Previous works showed that using ITP prior to CE separation could facilitate more than 2 orders of magnitude improvement in LOQ despite the presence of strong EOF. ${ }^{27,28}$ Our previous study also demonstrated that using ITP preconcentration could decrease the LOQ of DQAMmiR with ssDNA hybridization probes by 2 orders of magnitude (see Figure $2 \mathrm{Aa}$ for more details). ${ }^{29}$ This success inspired us to investigate if the same strategy would also work for DQAMmiR with PNA probes. However, our attempts to implement ITP for DQAMmiR with PNA probes failed because ITP could not concentrate unreacted PNA probes due to their very low electrophoretic mobilities (Figure 2Ab).$^{18}$ Consequently, the unreacted PNA probes eluted from the capillary during ITP, affecting quantitativeness of DQAMmiR, which utilizes the peak(s) of unreacted probes for normalizing the signals from the hybrids (Figure 1). Therefore, a new OSP technique that would be compatible with DQAMmiR utilizing PNA probes was needed.

Here, we introduce a new OSP technique based on the integration of FASS and ITP for DQAMmiR utilizing PNA 
probes. Although FASS is more sensitive to EOF in contrast to ITP,${ }^{26}$ it is a less mobility-selective method which can concentrate all negative charged species regardless of the magnitudes of their electrophoretic mobilities from a low-conductivity zone into a narrow zone at the interface with the high-conductivity zone due to an electric field-amplification phenomenon. ${ }^{20}$ Thus, with the addition of FASS to ITP (see Figure 2B for details), the nearneutral PNA probes in the low-conductivity sample plug could also be concentrated into a narrow zone along with the highmobility PNA-miRNA hybrids despite the probes' very low mobilities, preventing the probes from eluting from the capillary during the following ITP step. Meanwhile, due to the co-presence of TE and LE in the capillary, the hybrids could still be focused by ITP, which would lead to a significant improvement in LOQ for detecting miRNAs. Unlike the hybrids, the unreacted probes do not focus during ITP, but this is an advantage as it keeps peak heights of unreacted probes within the signal dynamic range tuned to detect low concentrations of the hybrids.

In this study, we first proved that the PNA probes were retained in the capillary during preconcentration by FASS-ITP, and, thus, that the FASS-ITP-CE approach could work for DQAMmiR utilizing PNA probes. An LOQ of $0.1 \mathrm{pM}$ was then demonstrated for accurately and precisely quantifying two miRNAs simultaneously, which was 140 times lower than that of the original approach without preconcentration. ${ }^{18} \mathrm{We}$ also showed that the FASS-ITP-CE approach was able to detect these miRNAs in crude cell lysates directly, indicating that the assay was robust to the presence of biological matrices. Although an LOQ of $0.1 \mathrm{pM}$ is still insufficient to detect all miRNAs in all samples, the 140-fold improvement in LOQ will largely increase the number of putative miRNAs fingerprints that can be validated by this assay. This improvement in LOQ brings DQAMmiR closer to becoming a practical tool for validation and clinical use of miRNA biomarkers of cancer.

\section{MATERIALS AND METHODS}

MiRNAs and PNA Probes. MiRNA targets and masking RNA were custom synthesized by IDT (Coralville, IA, USA). All PNA probes were custom synthesized by Panagene Inc. (Daejeon, South Korea). Detail information on miRNAs and PNA probes can be found in Note S2. Concentrations of miRNAs and PNA probes in stock solutions were determined using light absorbance at $260 \mathrm{~nm}$ measured with a NanoDrop ND-1000 spectrophotometer (Thermo-Fisher Scientific, Waltham, MA, USA); extinction coefficients were provided by respective suppliers of miRNA and PNA.

Hybridization Reactions. MiRNA-PNA hybridization was carried out in a Mastercycler 5332 thermocycler (Eppendorf, Hamburg, Germany). According to the information provided by the supplier of PNA, all the PNA-miRNA hybrids used in this study have melting temperatures $\left(T_{\mathrm{m}}\right)$ above $80^{\circ} \mathrm{C}$. Thus, we selected $60{ }^{\circ} \mathrm{C}$ as hybridization temperature. ${ }^{30}$ The temperature was first increased to a denaturing level of $95{ }^{\circ} \mathrm{C}$ and then lowered to $60^{\circ} \mathrm{C}$ at a rate of $20^{\circ} \mathrm{C} / \mathrm{min}$. After that the temperature was held at $60{ }^{\circ} \mathrm{C}$ for 30 min to allow hybridization.

CE-LIF Instrument. All experiments were performed using a P/ACE MDQ CE instrument (SCIEX, Brea, CA, USA) equipped with a laser-induced fluorescence detector. We used bare fusedsilica capillaries (Polymicro Technologies, Phoenix, AZ, USA) with an outer diameter of $365 \mu \mathrm{m}$, an inner diameter of $75 \mu \mathrm{m}$, and a total length of $80 \mathrm{~cm}$. The distance from the detector windows to the capillary inlet and outlet were 70 and $10 \mathrm{~cm}$, respectively (refer to Figure 2 for assignments of capillary inlet and outlet). New capillaries were preconditioned by flushing with methanol, $0.1 \mathrm{M} \mathrm{HCl}, 0.1 \mathrm{M} \mathrm{NaOH}$, deionized $\mathrm{H}_{2} \mathrm{O}$, and a $\mathrm{CE}$ running buffer (100 mM Tris-Cl, 20\% (v/v) acetonitrile (ACN),
$\mathrm{pH} 8.2)$ under a pressure of $20 \mathrm{psi}(138 \mathrm{kPa})$ for $30,30,60,30$, and $120 \mathrm{~min}$, respectively. Twenty percent $(\mathrm{v} / \mathrm{v})$ of $\mathrm{ACN}$ was added into the running buffer to improve the solubility of PNA probes. ${ }^{18}$ The capillary was rinsed prior to every run with $0.1 \mathrm{M}$ $\mathrm{HCl}, 0.1 \mathrm{M} \mathrm{NaOH}$, deionized $\mathrm{H}_{2} \mathrm{O}$, and the $\mathrm{CE}$ running buffer for $1 \mathrm{~min}$ each under a 20-psi pressure. The capillary coolant temperature was kept at $20^{\circ} \mathrm{C}$ during all $\mathrm{CE}$ experiments. Fluorescence of the Alexa Fluor 647 dye on the PNA probes was excited by $638 \mathrm{~nm}$ light generated with a continuous-wave solid state laser (Pavilion Integration Group, San Jose, CA, USA) and detected at $679 \mathrm{~nm}$ using an emission filter setup composed of a $655 \mathrm{~nm}$ long-pass filter (SCIEX, Brea, CA, USA) and a 679/41 nm bandpass filter (Semrock, Rochester, NY, USA).

Standard CE-Based DQAMmiR. The hybridization mixture was injected into the capillary by a pressure pulse of $0.5 \mathrm{psi}$ $(3.4 \mathrm{kPa})$ for $5 \mathrm{~s}$ from the capillary inlet. Electrophoresis was driven by an electric field of $375 \mathrm{~V} / \mathrm{cm}$ with a positive polarity at the sample-injection end. Electropherograms were analyzed with 32 Karat Software (SCIEX, Brea, CA, USA).

FASS-ITP-CE for DQAMmiR. The capillary was prefilled with the TE buffer (20 mM Tris-HEPES, pH 8.2) with a pressure of 20 psi $(137.9 \mathrm{kPa})$ for $1 \mathrm{~min}$. The sample in a low-conductivity buffer ( $1 \mathrm{mM}$ Tris-Cl, $20 \%$ (v/v) ACN, pH 8.2) was injected from the capillary outlet by a pressure pulse of $3 \mathrm{psi}(20.7 \mathrm{kPa})$ for $99 \mathrm{~s}$, which gives a sample plug length of $44.9 \mathrm{~cm}$. A LE buffer of $100 \mathrm{mM}$ Tris-Cl, 20\% (v/v) ACN, pH 8.2 was used in the vial at the capillary outlet while the inlet end was kept in a vial containing TE buffer; an electric field of $375 \mathrm{~V} / \mathrm{cm}$ was applied with positive polarity at the capillary outlet to facilitate FASS-ITP preconcentration as described in Figure 2B. The FASS-ITP was stopped when the concentrated analytes reach near the capillary inlet by turning off the voltage. The stop time for the FASS-ITP step was determined by observing the electrical current profile of the run (see Note S3). The buffer at the capillary inlet was then replaced with LE and an electric field of $375 \mathrm{~V} / \mathrm{cm}$ was applied with positive polarity at the capillary inlet to resume $\mathrm{CE}$ separation. The unreacted PNA probes and the PNA-miRNA hybrids were then separated by CE and detected with LIF as in the standard DQAMmiR approach. ${ }^{18}$

Quantitation of miRNA. Peak areas were divided by the corresponding migration times to compensate for the area dependence on the species residence time while its passing the capillary detection window with different velocities. Concentrations of miRNAs were determined using the following equation: ${ }^{18}$

$$
[\mathrm{miRNA}]^{i}=\frac{A_{\mathrm{H}}^{i}}{q_{\mathrm{H}}^{i} q_{\mathrm{P}}^{i}} \frac{\sum_{i=1}^{N}\left(q_{\mathrm{P}}^{i}[\mathrm{P}]_{0}^{i}\right)}{A_{\mathrm{P}}+\sum_{i=1}^{N} \frac{A_{\mathrm{H}}^{i}}{q_{\mathrm{H}}^{i}}}
$$

where $[\mathrm{P}] 0^{i}$ is the total concentration of the $i$-th PNA probe (composed of the hybrid and the excess probe), $A_{\mathrm{H}^{i}}$ is the area corresponding to the $i$-th hybrid, $A \mathrm{P}$ is the cumulative area of the unbound probes, $q \mathrm{P}^{i}$ is a relative quantum yield of the $i$-th PNA probe to normalize the quantum yield differences between the probes, and $q_{\mathrm{H}}{ }^{i}$ is the relative quantum yield of the $i$-th hybrid with respect to that of the unbound probe. These relative quantum yields, $q \mathrm{P}^{i}$ and $q_{\mathrm{H}}{ }^{i}$, were found experimentally as described in Note S4.

\section{RESULTS AND DISCUSSION}

The insufficiently low LOQ of CE-LIF-based DQAMmiR limits its utility for validation of miRNA fingerprints. This issue could be potentially resolved by incorporating OSP techniques, such as ITP, prior to CE separation. Our previous study demonstrated that using ITP preconcentration before $\mathrm{CE}$ 
separation could decrease LOQ of DQAMmiR with ssDNA probes by two orders of magnitudes, which inspired us to investigate whether the same ITP-CE strategy would be applicable to DQAMmiR with PNA hybridization probes.

In ITP, analytes can be concentrated only if they have electrophoretic mobilities greater than that of the TE ion and lower than that of the LE ion. ${ }^{24}$ Because the miRNA quantitation in DQAMmiR requires accurate detection of both unreacted probes and the miRNA-probe hybrids, ${ }^{18}$ it is essential to have TE/LE chemistry that can concentrate both the PNA probes and the hybrids in ITP-CE for DQAMmiR with PNA probes. Thus, the following conditions need to be satisfied for electrophoretic mobilities:

$$
\left|\mu_{\mathrm{TE}}\right|<\left|\mu_{\mathrm{PNA}}\right|<\left|\mu_{\text {hybrid }}\right|<\left|\mu_{\mathrm{LE}}\right|
$$

where $\mu_{\mathrm{TE}}, \mu_{\mathrm{PNA}}, \mu_{\text {hybrid}}$, and $\mu_{\mathrm{LE}}$ are mobilities of the TE ion, PNA probes, PNA-miRNA hybrids, and the LE ion, respectively. However, the mobilities of PNA probes are near zero $\left(\left|\mu_{\mathrm{PNA}}\right| \approx 0\right)$ due to their electrically neutral backbones. Only the C-terminus and a fluorescent dye provide a small negative charge resulting in small net electrophoretic mobilities for PNA probes, making it very challenging to find a suitable TE ion to satisfy inequalities in eq 2. A previous study by Bercovici et al. demonstrated that the PNA probes could not be focused by ITP where they used 2morpholin-4-ium-4-ylethanesulfonate ion (MES) as a TE ion and $\mathrm{Cl}^{-}$as an LE ion. ${ }^{31}$

In order to investigate the feasibility of implementing ITP-CE for DQAMmiR with PNA probes, we also experimentally tested another two common TE ions: 2-[4-(2-hydroxyethyl)piperazin-1yl]ethanesulfonic ion (HEPES) and Tricine, which have smaller net mobilities than MES according to the AnglerFish software, ${ }^{32}$ while using the high-mobility $\mathrm{Cl}^{-}$ion as the LE ion. In these experiments, a hybridization mixture of two miRNA targets, miR$20 \mathrm{~b}$ and miR-100, and their complementary PNA probes, named here as PNA-20b and PNA-100, taken in excess was prepared and subjected to ITP-CE (see Note S5). The results (Figure 3) showed that the peaks for the unreacted PNA probes either were unexpectedly small or disappeared in experiments using either of the two different TE ions despite the PNA probes being more than ten times in excess of miRNAs in the hybridization mixture. These results proved that the unreacted PNA probes eluted from the capillary during sample preconcentration by ITP, indicating that net electrophoretic mobilities of both tested TE ions were larger than that of the PNA probes. Since the quantitation of miRNAs in DQAMmiR requires detection of not only the hybrids but also the unreacted probes, ITP as a sole preconcentration method is not compatible with DQAMmiR utilizing PNA probes. Therefore, we had to find a new OSP technique that could decrease the LOQ of DQAMmiR while utilizing PNA probes.

To address this issue, we explored other alternative OSP techniques and realized that integrating FASS with ITP could be a potential solution to preconcentrate the hybrids efficiently while preventing the loss of the unreacted PNA probes. In contrast to ITP, FASS is a less mobility-selective process for sample concentration, i.e. it can concentrate all negative or positive charged species regardless of the magnitude of their mobilities from a low-conductivity plug into a narrow zone at the interfaces with a high-conductivity zone due to an electric fieldamplification effect. ${ }^{20}$ As described in Figure 2B, the FASS-ITP$\mathrm{CE}$ approach can be implemented by preparing the hybridization in an extremely diluted buffer with the conductivity significantly lower than that of both TE and LE. Thus, an amplified electric field can be facilitated in the sample plug once the high voltage is applied, leading to concentration of both the low-mobility PNA probes and the high-mobility PNA-miRNA hybrids at the sample/LE interface by FASS. On the other hand, a diffused TE zone is also formed by the counter broadening effect at the

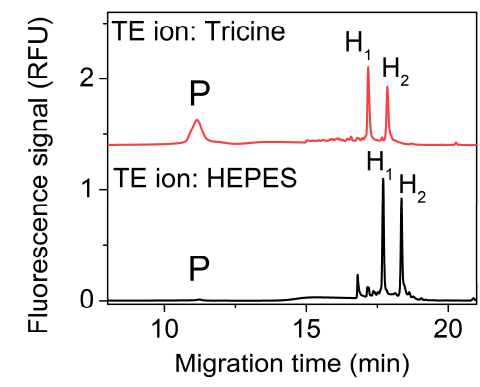

Figure 3. Investigation of two TE ions - Tricine and HEPES - in ITPCE for DQAMmiR with PNA hybridization probes. A hybridization mixture of $0.1 \mathrm{nM}$ miR-20b, $0.1 \mathrm{nM}$ miR-100, $5 \mathrm{nM}$ PNA-20b, and $1 \mathrm{nM}$ PNA-100 were prepared in the LE buffer composed of $100 \mathrm{mM}$ Tris-Cl, $20 \%(\mathrm{v} / \mathrm{v}) \mathrm{ACN}, \mathrm{pH} 8.2$ and analyzed by ITP-CE. The TE buffers composed of $10 \mathrm{mM}$ Tris, $50 \mathrm{mM}$ Tricine, $\mathrm{pH} 7.3$ (top trace) and $20 \mathrm{mM}$ Tris, $10 \mathrm{mM}$ HEPES, pH 8.2 (bottom trace) were used in these experiments, respectively. $\mathrm{P}$ stands for excess PNA probes; $\mathrm{H}_{1}$ stands for the PNA-miR-100 hybrid; and $\mathrm{H}_{2}$ stands for the PNA-miR-20b hybrid.

TE/sample plug boundary. As a result, a discontinued TEanalytes-LE pattern, which is required for ITP, is formed in the capillary. Therefore, the PNA-miRNA hybrids would be concentrated at the TE/LE interface into a narrower band as the condition of $|\mu \mathrm{TE}|<\left|\mu_{\text {hybrid }}\right|<\left|\mu_{\mathrm{LE}}\right|$ is satisfied. In theory, the stacked PNA probes should be slowly separated from the ITPfocused hybrids because their net mobilities are smaller than that of the TE ion. However, this separation of the stacked PNA probes from the ITP-focused hybrids would be limited because the electric field in the analyte zone at the TE/LE interface is low due to the presence of the low-conductivity diluted TE zone in the capillary. Thus, the unreacted PNA probes would be kept along with the concentrated hybrids in the capillary for the subsequent CE separation after reversing the polarity, which would allow us to facilitate accurate quantitation of miRNAs like in the standard CE-based DQAMmiR.

In order for this FASS-ITP-CE approach to work, four requirements must be satisfied: 1) PNA probes must hybridize with their miRNA targets in the low-concentration buffer as a low-conductivity sample plug is required for FASS-ITP-CE; 2) unreacted PNA probes must be kept inside the capillary during the sample preconcentration process, as they are essential for accurate miRNA quantitation; 3) PNA-miRNA hybrids must be concentrated efficiently to achieve low LOQ for analyzing miRNAs in cell lysates; 4) the assay must be suitable for quantifying multiple miRNAs with high accuracy and precision.

As FASS-ITP requires a sample plug with low conductivity, it is essential to investigate if PNA probes can still bind their miRNA targets tightly in low-concentration buffers. It is known that low-concentration buffers are typically not favored by nucleic acid hybridization. ${ }^{33}$ However, thanks to the neutral backbone of PNA, PNA-RNA duplexes are much more stable than the DNARNA duplexes in low-concentration buffers due to the lack of coulomb repulsion forces between the two strands. ${ }^{32}$ Relative neutrality of PNA with respect to DNA should make it possible to perform PNA-miRNA hybridization reactions in lowconcentration buffers. To confirm this, we prepared hybridization mixtures in Tris-Cl buffers with concentrations of 100, 10, and $1 \mathrm{mM}$. These hybridization mixtures were analyzed by standard CE-based DQAMmiR as described in Materials and Methods. The results in Figure 5A show that the separation of unreacted PNA probes from the hybrids and the hybrids from each other was not affected by the low concentrations of the sample buffer, which allowed us to facilitate DQAMmiR in these buffers. Quantitation results from triplicated measurements in Figure 5B 
A

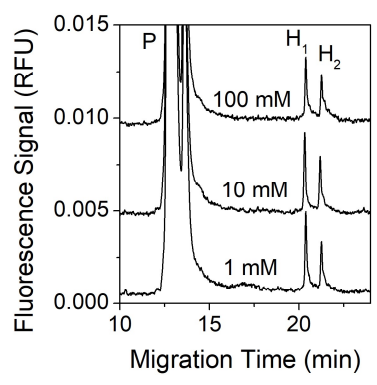

B

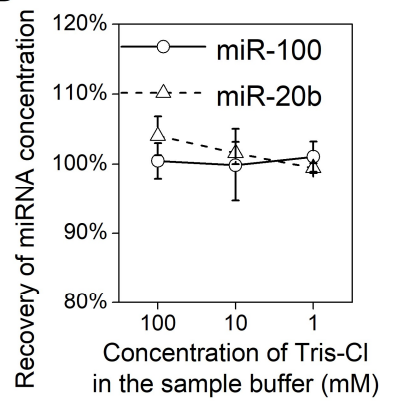

Figure 5. The effect of sample buffer concentration on PNA-miRNA hybridization. A hybridization mixture of $50 \mathrm{pM}$ miR-20b, $50 \mathrm{pM}$ miR100, $5 \mathrm{nM}$ PNA-20b, and $1 \mathrm{nM}$ PNA-100 were prepared in buffers composed of 1 to $100 \mathrm{mM}$ Tris- $\mathrm{Cl}, 20 \%(\mathrm{v} / \mathrm{v}) \mathrm{ACN}$, at $\mathrm{pH} 8.2$. They were then analyzed by standard CE-based DQAMmiR. Electropherograms (A) and the quantitation results (B) are shown here. P stands for excess PNA probes; $\mathrm{H}_{1}$ stands for the PNA-miR-100 hybrid; and $\mathrm{H}_{2}$ stands for PNAmiR-20b hybrid. Error bars represent one standard deviation from three independent runs.

showed recoveries of $95 \%$ to $105 \%$ for measuring two miRNAs in all samples with RSD of less than $5 \%$. It indicates that the concentration of the sample buffer has negligible effect on PNAmiRNA hybridization. PNA probes could still bind their complementary miRNA targets tightly in a very diluted buffer of $1 \mathrm{mM}$ Tris- $\mathrm{Cl}, 20 \%(\mathrm{v} / \mathrm{v}) \mathrm{ACN}, \mathrm{pH} 8.2$, which would qualify for the low-conductivity sample buffer required in FASS-ITP.

We then had to prove that combining FASS with ITP would prevent the loss of the low-mobility PNA probes in the preconcentration process. As any loss of PNA probes would result in decreasing of their fluorescence signal in the electropherogram, we designed experiments in which the same sample containing only PNA probes would be analyzed by FASS-ITP-CE and CE in parallel. Thus, we could evaluate if FASS-ITP-CE retained the PNA probes by comparing the fluorescence signal of the PNA probes obtained from FASS-ITP-CE with that obtained from CE (which would be used as a reference). In this FASS-ITP-CE experiment, two buffers, $20 \mathrm{mM}$ Tris-HEPES at $\mathrm{pH} 8.2$ and $100 \mathrm{mM}$ Tris-Cl with $20 \%(\mathrm{v} / \mathrm{v}) \mathrm{ACN}$ at $\mathrm{pH} 8.2$, were used as TE and LE, respectively. The diluted buffer composed of $1 \mathrm{mM}$ Tris$\mathrm{Cl}$ and $20 \%(\mathrm{v} / \mathrm{v}) \mathrm{ACN}$ at $\mathrm{pH} 8.2$, which has the conductivity much lower than those of both TE and LE buffer, was used as sample buffer. The sample was analyzed by FASS-ITP-CE as described in Materials and Methods. In parallel, another sample with the same concentrations of PNA probes was prepared in LE and analyzed by $\mathrm{CE}$ with $\mathrm{LE}$ as a running buffer. In this $\mathrm{CE}$ experiment, a $44.9-\mathrm{cm}-$ long plug of the sample was injected by pressure; the same plug length was used in FASS-ITP-CE. Thus, the quantities of PNA probes injected into the capillary were identical in these two runs. Because the running buffer in this experiment was LE and was identical to the running buffer in FASS-ITP-CE. The velocities of the PNA probes passing through the detector were also identical in these two runs. Therefore, we could examine if the PNA probes were retained in FASS-ITP-CE by directly comparing the peak area of the PNA probes with that obtained from the CE run. A ratio between peak areas in FASSITP-CE and CE runs was equal to 1.03 (Figure 4), suggesting that the PNA probes were kept inside the capillary in the FASS-ITP preconcentration process. The $3 \%$ difference results from errors in the volume of injected sample. This excellent recovery proves that the combination of FASS with ITP prevents the unreacted PNA probes from eluting from the capillary (as expected). Retaining the probes, in turn, allows us to perform accurate miRNA quantitation after CE separation of the hybrids from each other and the unreacted probes and LIF detection of all these

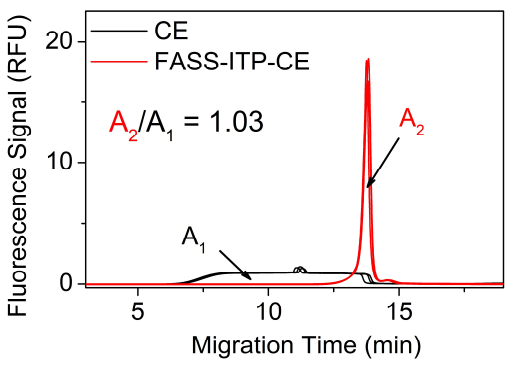

Figure 4. Proof of the ability to retain PNA probes in FASS-ITP-CE. A mixture of $5 \mathrm{nM}$ PNA-20b and $1 \mathrm{nM}$ PNA-100 were prepared and analyzed by CE (black traces with an average peak area of $A_{1}$ ) and FASSITP-CE (red traces with an average peak area of $\mathrm{A}_{2}$ ). Samples were injected by pressure of 3 psi for $99 \mathrm{~s}$ in both cases. Three repeated runs of each experiment shown here indicate good reproducibility.

analytes.

We also evaluated the efficiency of FASS-ITP-facilitated preconcentration of the PNA-miRNA hybrids. As shown in Figure 6A, the separation of the unreacted PNA probes from the hybrids and two PNA-miRNA hybrids from each other in DQAMmiR were not affected by the additional preconcentration step. Very sharp peaks were detected for both hybrids, producing peaks with heights 100 times greater than those obtained without preconcentration, suggesting that the LOQ of FASS-ITP-CE could be more than 2 orders of magnitude lower than that of the standard CE for detecting miRNAs. It was also noticed that these

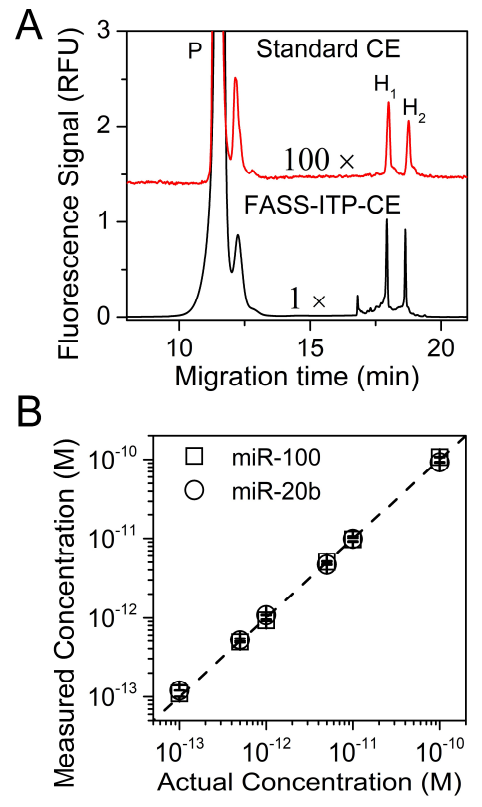

Figure 6. Evaluation of the preconcentration efficiency and the analytical performances of FASS-ITP-CE. A) Electropherograms for measurements by standard CE and FASS-ITP-CE for DQAMmiR. A hybridization mixture of $0.1 \mathrm{nM}$ miR-20b, $0.1 \mathrm{nM}$ miR-100, $5 \mathrm{nM}$ PNA-20b and $1 \mathrm{nM}$ PNA-100 were analyzed by the standard CE approach and the FASS-ITP$\mathrm{CE}$ approach in parallel. The trace of the standard CE approach was zoomed in by a factor of 100 along the vertical axis for its easy comparison with the FASS-ITP-CE trace. P stands for unreacted PNA probes; $\mathrm{H}_{1}$ stands for the PNA-miR-100 hybrid; and $\mathrm{H}_{2}$ stands for the PNA-miR-20b hybrid. B) Quantification of the two miRNAs, miR-20b and miR-100, by the FASS-ITP-CE approach in a concentration range of 0.1 to $100 \mathrm{pM}$. Measured concentrations are plotted with respect to their actual concentrations, determined by UV absorbance at $260 \mathrm{~nm}$. The dashed line $(y=x)$ represents the recovery of $100 \%$. The error bars represent one standard deviation of three independent replications. 
peak heights were comparable with those in Figure 3, indicating that the combination of FASS with ITP provided preconcentration efficiency similar to that of ITP alone for concentrating the PNAmiRNA hybrids. It's worth noticing that a previous study reported that the combination of ITP with FASS in a coated capillary (with suppressed EOF) could provide a preconcentration efficiency higher than that of ITP alone. ${ }^{35}$ In our case of an uncoated capillary with strong EOF, the addition of FASS to ITP didn't further improve the efficiency of concentrating the PNA-miRNA hybrids. Most importantly, however, FASS did not interfere with the concentration efficiency of ITP for the PNA-miRNA hybrids and could still greatly decrease the LOQ of the assay for detecting multiple miRNAs.

The analytical performance of the FASS-ITP-CE assay was then evaluated by quantifying miR-20b and miR-100 with varying concentrations. The electropherograms from these experiments are shown in Note S7. The quantitation results (Figure 6B) demonstrate that the miRNA concentrations measured by the FASS-ITP-CE approach agree well with the known concentrations of the spiked-in targets in a concentration range of 0.1 to $100 \mathrm{pM}$, giving recoveries within a range of 90 to $110 \%$ with $\mathrm{RSD} \leq 10 \%$ for all concentrations. These experiments revealed that the FASS-ITP-CE approach was applicable to DQAMmiR utilizing PNA probes. The results show that FASSITP-CE can quantify two miRNAs accurately and precisely with LOQ of $0.1 \mathrm{pM}$, which is 140-times lower than that of the standard approach as reported in the previous study. ${ }^{18}$ Although an LOQ of $0.1 \mathrm{pM}$ is still insufficient to detect low-abundance miRNAs, the 140-times improvement in LOQ will facilitate validation of putative miRNAs fingerprints with a greater variability in miRNA concentrations in the sample.

Finally, the feasibility of FASS-ITP-CE for analyzing miRNAs in a biological matrix was assessed. We analyzed two endogenous miRNAs (miR-100 and miR-20b) in two cell lines: a prostate cancer-derived cell line, 22Rv1, and a normal prostate cell line, RWPE-1. In these experiments, measurements were conducted by determining the miRNA concentrations in the crude cell lysate directly, i.e., the cultured cells were lysed and analyzed without additional sample purification (see Notes S8 and S9). The results (Figure 7) show that both miR-100 and miR-20b were successfully detected in the $22 \mathrm{Rv} 1$ cell lysate, while only miR100 was detectable by the current assay in the RWPE-1 cell lysate. It revealed that the expression level of miR-20b is greater in the cancer cells than in the normal cells, while the expression levels of miR-100 were similar in the cancer and normal cells (Note S9). This result agrees well with the previous report of miR-20b being an oncogene in prostate cancer. ${ }^{36}$ Known concentrations of the two miRNAs were also spiked into cell lysates to account for any effect the cell contents could have on quantification; the effect was found to be negligible (Note S9). This example of directly measuring two miRNAs simultaneously in crude cell lysates indicates that our FASS-ITP-CE approach is not affected by the contents of the sample matrix, thus, suggesting FASS-ITP-CE as a practical tool for validation of some putative miRNA fingerprints in patient samples.

\section{CONCLUSIONS}

In this study, we introduce a sample preconcentration technique for DQAMmiR utilizing PNA probes for analysis of multiple miRNAs. This preconcentration technic combines FASS with ITP prior to $\mathrm{CE}$ to greatly decrease LOQ of DQAMmiR. We demonstrated that FASS-ITP could efficiently concentrate PNAmiRNA hybrids while preventing the loss of PNA probes, proving that FASS-ITP-CE is applicable to DQAMmiR utilizing PNA probe in principle. Using FASS-ITP-CE for simultaneous analysis of two miRNAs showed that an LOQ of $0.1 \mathrm{pM}$ could be

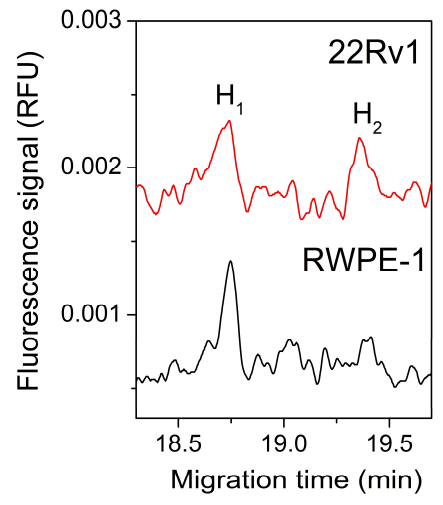

Figure 7. FASS-ITP-CE analysis of miR-100 and miR-20b in $22 \mathrm{Rv} 1$ cell lysate (top trace) and RWPE-1 cell lysate (bottom trace). $\mathrm{H}_{1}$ marks peaks of the PNA-miR-100 hybrid and $\mathrm{H}_{2}$ marks the peak of the PNA-miR-20b hybrid.

achieved. This LOQ is about 140 times lower than that of the standard CE-based DQAMmiR. We also showed that FASS-ITPCE was suitable for analyzing endogenous miRNAs in crude cell lysates directly, suggesting that the method was robust to variations in the sample-matrix components. Although, an LOQ of $0.1 \mathrm{pM}$ is still insufficient to detect low-abundance miRNAs, lowering LOQ by a factor of 140 and methods robustness to sample-matrix contents will largely expand the utility of DQAMmiR, bringing it closer to becoming a practical tool for validation and clinical use of miRNA biomarkers.

\section{ASSOCIATED CONTENT}

\section{Supporting Information}

The Supporting Information is available free of charge at Note S1, estimation of the concentration levels of miRNA targets in clinical samples; Note S2, sequences of the miRNA targets and their complementary PNA probes; Note $\mathrm{S} 3$, determination of the stop time, $t_{\mathrm{st}}$, for the FASS-ITP step in FASS-ITP-CE; Note S4, relative quantum yields measurements; Note S5, ITP-CE for DQAMmiR; Note S6, purification of PNA probes; Note S7, electropherograms and results of miRNA quantification by FASS-ITP-CE; Note S8, protocol of cell culture. Note S9, analyzing miRNAs in crude cell lysates (PDF)

\section{AUTHOR INFORMATION}

\section{Corresponding Author}

*E-mail: skrylov@yorku.ca.

\section{ORCID}

Liang Hu: 0000-0003-4507-8525

Svetlana M. Krylova: 0000-0002-3291-6721

Stanley K. Liu: 0000-0001-6851-0857

George M. Yousef: 0000-0003-3859-779X

Sergey N. Krylov: 0000-0003-3270-2130

\section{Author Contributions}

All authors have given approval to the final version of the manuscript.

\section{Notes}

The authors declare no competing financial interest. 


\section{ACKNOWLEDGMENTS}

The authors thank Natural Sciences and Engineering Research Council of Canada (Discovery Grant number 238990) for financial support.

\section{REFERENCES}

(1) He, L.; Hannon, G. J. MicroRNAs: Small RNAs with a Big Role in Gene Regulation. Nat. Rev. Genet. 2004, 5, 522-531.

(2) Chen, P.; Su, J.; Hung, M. Dysregulation of microRNAs in Cancer. J. Biomed. Sci. 2012, 19, 90.

(3) Guerau-de-Arellano, M.; Alder, H.; Ozer, H. G.; LovettRacke, A.; Racke, M. K. miRNA Profiling for Biomarker Discovery in Multiple Sclerosis: from Microarray to Deep Sequencing. J. Neuroimmunol. 2012, 248, 32-39.

(4) Lopez, J. P.; Diallo, A.; Cruceanu, C.; Fiori, L. M.; Laboissiere, S.; Guillet, I.; Fontaine, J.; Ragoussis, J.; Benes, V.; Turecki, G.; Ernst, C. Biomarker Discovery: Quantification of MicroRNAs and Other Small Non-Coding RNAs Using Next Generation Sequencing. BMC Medical Genom. 2015, 8, 35.

(5) Lyu, J.; Zhao, L.; Wang, F.; Ji, J.; Cao, Z.; Xu, H.; Shi, X.; Zhu, Y.; Zhang, C.; Guo, F.; Yang, B.; Sun, Y. Discovery and Validation of Serum microRNAs as Early Diagnostic Biomarkers for Prostate Cancer in Chinese Population. Biomed. Res. Int. 2019, 2019, 9306803.

(6) He B.; Zhao Z.; Cai Q.; Zhang Y.; Zhang P.; Shi S.; Xie H.; Peng X.; Yin W.; Tao Y.; Wang X. miRNA-Based Biomarkers, Therapies, and Resistance in Cancer. Int. J. Biol. Sci. 2020, 16, 2628-2647.

(7) Schmidt, L.; Fredsøe, J.; Kristensen, H.; Strand, S. H.; Rasmussen, A.; Høyer, S.; Borre, M.; Mouritzen, P.; Ørntoft, T.; Sørensen, K. D. Training and Validation of a Novel 4-miRNA Ratio Model (MiCaP) for Prediction of Postoperative Outcome in Prostate Cancer Patients. Ann. Oncol. 2018, 29, 2003-2009.

(8) Bustin, S. A.; Nolan, T. Pitfalls of Quantitative Real-Time Reverse-Transcription Polymerase Chain Reaction. J. Biomol. Tech. 2004, 15, 155-166.

(9) Bleker de Oliveira, M.; Koshkin, V.; Liu, G.; Krylov, S. N. Analytical Challenges in Development of Chemoresistance Predictors for Precision Oncology. Anal. Chem. 2020, 92, published ASAP. DOI: 10.1021/acs.analchem.0c02644.

(10) Labib, M.; Khan, N.; Ghobadloo, S. M.; Cheng, J.; Pezacki, J. P.; Berezovski, M. V. Three-Mode Electrochemical Sensing of Ultralow MicroRNA Levels. J. Am. Chem. Soc. 2013, 135, 3027-3038.

(11) Yang, C.; Dou, B.; Shi, K.; Chai, Y.; Xiang, Y.; Yuan, R. Multiplexed and Amplified Electronic Sensor for the Detection of microRNAs from Cancer Cells. Anal. Chem. 2014, 86, 11913-11918.

(12) Qavi, A. J.; Bailey, R. C. Multiplexed Detection and Label-Free Quantitation of MicroRNAs Using Arrays of Silicon Photonic Microring Resonators. Angew. Chem. Int. Ed. 2010, 49, 4608-4611.

(13) Lee, H.; Shapiro, S. J.; Chapin, S. C.; Doyle, P. S. Encoded Hydrogel Microparticles for Sensitive and Multiplex microRNA Detection Directly from Raw Cell Lysates. Anal. Chem. 2016, 88, 3075-3081.

(14) Xu, F.; Yang, T.; Chen, Y. Quantification of microRNA by DNA-Peptide Probe and Liquid Chromatography-Tandem Mass Spectrometry-Based Quasi-Targeted Proteomics. Anal. Chem. 2016, 88, 754-763.

(15) Roy, S.; Soh, J. H.; Gao, Z. A Microfluidic-Assisted Microarray for Ultrasensitive Detection of miRNA under an Optical Microscope. Lab Chip 2011, 11, 1886-1894.

(16) Cheng, Y.; Dong, L.; Zhang, J.; Zhao, Y.; Li, Z. Recent Advances in microRNA Detection. Analyst 2018, 143, $1758-1774$.
(17) Wegman, D. W.; Krylov, S. N. Direct Quantitative Analysis of Multiple miRNAs (DQAMmiR). Angew. Chem. Int. Ed. 2011, 50, 10335-10339.

(18) Hu, L.; Anand, M.; Krylova, S. M.; Yang, B. B.; Liu, S. K.; Yousef, G. M.; Krylov, S. N. Direct Quantitative Analysis of Multiple microRNAs (DQAMmiR) with Peptide Nucleic Acid Hybridization Probes. Anal. Chem. 2018, 90, 14610-14615.

(19) Hu, L.; Stasheuski, A. S.; Wegman, D. W.; Wu, N.; Yang, B. B.; Hayder, H.; Peng, C.; Liu, S. K.; Yousef, G. M.; Krylov, S. N. Accurate MicroRNA Analysis in Crude Cell Lysate by Capillary Electrophoresis-Based Hybridization Assay in Comparison with Quantitative Reverse Transcription-Polymerase Chain Reaction. Anal. Chem. 2017, 89, 4743-4748.

(20) Burgi, D. S.; Chien, R. L. Optimization in Sample Stacking for High-Performance Capillary Electrophoresis. Anal. Chem. 1991, 63, 2042-2047.

(21) Chien, R. L.; Burgi, D. S. Sample Stacking of an Extremely Large Injection Volume in High-Performance Capillary Electrophoresis. Anal. Chem. 1992, 64, 1046-1050.

(22) Monton, M. R.; Imami, K.; Nakanishi, M.; Kim, J. B.; Terabe, S. Dynamic pH Junction Technique for On-Line Preconcentration of Peptides in Capillary Electrophoresis. $J$. Chromatogr. A 2005, 1079, 266-273.

(23) Quirino, J. P.; Kim, J. -B.; Terabe, S. Sweeping: Concentration Mechanism and Applications to High-Sensitivity Analysis in Capillary Electrophoresis. J. Chromatogr. A 2002, 965, 357-373.

(24) Stegehuis, D. S.; Irth, H.; Tjaden, U. R.; Van der Greef, J. Isotachophoresis as an On-Line Concentration Pretreatment Technique in Capillary Electrophoresis. J. Chromatogr. 1991, 538, 393-402.

(25) Hirokawa, T.; Ohmori, A.; Kiso, Y. Analysis of a Dilute Sample by Capillary Zone Electrophoresis with Isotachophoretic Preconcentration. J. Chromatogr. 1993, 634, 101-106.

(26) Bharadwaj, R.; Santiago, J. G. Dynamics of FieldAmplified Sample Stacking. J. Fluid Mech. 2005, 543, 57-92.

(27) Adam, T.; Sevcík, J.; Svagera, Z.; Fairbanks, L. D.; Barták, P. Determination of Adenosine Deaminase Activity in Human Erythrocytes by On-Column Capillary IsotachophoresisCapillary Zone Electrophoresis in the Presence of Electroosmotic Flow. Electrophoresis 1999, 20, 564-568.

(28) Riaz, A.; Chung, D. S. Transient Isotachophoresis of Highly Saline Trace Metals under Strong Electroosmotic Flow Conditions. Electrophoresis 2005, 26, 668-673.

(29) Wegman, D. W.; Ghasemi, F.; Khorshidi, A.; Yang, B. B.; Liu, S. K.; Yousef, G. M.; Krylov, S. N. Highly-Sensitive Amplification-Free Analysis of Multiple miRNAs by Capillary Electrophoresis. Anal. Chem. 2015, 87, 1404-1410.

(30) Weiler, J.; Gausepohl, H.; Hauser, N.; Jensen, O. N.; Hoheisel, J. D. Hybridisation Based DNA Screening on Peptide Nucleic Acid (PNA) Oligomer Arrays. Nucleic Acids Res. 1997, 25, 2792-2799.

(31) Ostromohov, N.; Schwartz, O.; Bercovici, M. Focused upon Hybridization: Rapid and High Sensitivity Detection of DNA Using Isotachophoresis and Peptide Nucleic Acid Probes. Anal. Chem. 2015, 87, 9459-9466.

(32) Malý, M.; Boublík, M.; Pocrnić, M.; Ansorge, M.; Lorinčíková, K.; Svobodová, J.; Hruška, V.; Dubský, P.; Gaš, B. Determination of Thermodynamic Acidity Constants and Limiting Ionic Mobilities of Weak Electrolytes by Capillary Electrophoresis Using a New Free Software AnglerFish. Electrophoresis 2020, 41, 493-501.

(33) Owczarzy, R.; You, Y.; Moreira, B. G.; Manthey, J. A.; Huang, L.; Behlke, M. A.; Walder, J. A. Effects of Sodium Ions on DNA Duplex Oligomers: Improved Predictions of Melting Temperatures. Biochemistry 2004, 43, 3537-3554. 
(34) Chim, W.; Sedighi, A.; Brown, C. L.; Pantophlet, R.; Li, P. C. H. Effect of Buffer Composition on PNA-RNA Hybridization Studied in the Microfluidic Microarray Chip. Can. J. Chem. 2018, 96, 241-247.

(35) Kawai, T.; Ota, N.; Imasato, A.; Shirasaki, Y.; Otsuka, K.; Tanaka, Y. Profiling of N-Linked Glycans from 100 Cells by Capillary Electrophoresis with Large-Volume Dual
Preconcentration by Isotachophoresis and Stacking. $J$. Chromatogr. A 2018, 1565, 138-144.

(36) Guo, J., Xiao, Z., Yu, X., Cao, R. miR-20b Promotes Cellular Proliferation and Migration by Directly Regulating Phosphatase and Tensin Homolog in Prostate Cancer. Oncol. Lett. 2017, 14, 6895-6900. 


\section{SUPPORTING INFORMATION}

\section{Necessity and Challenges of Sample Preconcentration in Analysis of Multiple MicroRNAs by Capillary Electrophoresis}

Liang Hu, ${ }^{1}$ Svetlana M. Krylova, ${ }^{1}$ Stanley K. Liu, ${ }^{2}$ George M. Yousef, ${ }^{3}$ and Sergey N. Krylov*

${ }^{1}$ Department of Chemistry and Centre for Research on Biomolecular Interactions, York University, Toronto, Ontario M3J 1P3, Canada

2 Department of Radiation Oncology, Sunnybrook-Odette Cancer Centre, 2075 Bayview Avenue, Toronto, Ontario M4N 3M5, Canada

${ }^{3}$ Department of Pediatric Laboratory Medicine, Hospital for Sick Children, 555 University Avenue, Toronto, Ontario M5G 1X8, Canada

\section{Table of Contents}

Page

Number

Note S1. Estimation of the concentration range of miRNA targets in clinical samples . . S S-2

Note S2. Sequences of miRNA targets and their PNA probes ............... S-2

Note S3.

Determination of the stop time, tst, for the FASS-ITP step in FASS-ITP-CE . .

Note S4. Relative quantum yields measurements $\ldots \ldots \ldots \ldots \ldots \ldots \ldots \ldots \ldots \ldots$ S-4

Note S5. Relative quantum yields measurements $\ldots \ldots \ldots \ldots \ldots \ldots \ldots \ldots \ldots \ldots$ S-4

Note S6. Purification of the PNA probes $\ldots \ldots \ldots \ldots \ldots \ldots \ldots \ldots \ldots \ldots \ldots \ldots \ldots \ldots \ldots \ldots \ldots$, S-5

Note S7. Electropherograms and results of miRNA quantification by FASS-ITP-CE ..... S-7

Note S8.

Protocol of cell culture . . . . . . . . . . . . . . . . . . . . . . . . . . . . . .

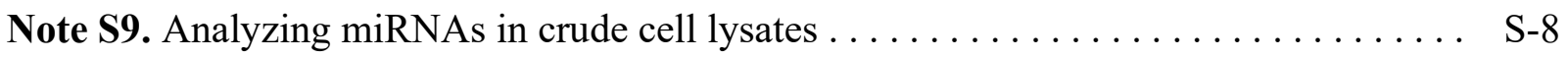

\section{Additional supplementary files}

File name........ Description/Experiment

raw data.zip ....... raw data (MS Excel)

figure data.zip ...... figure files (Origin and Adobe Illustrator) 


\section{Note S1. Estimation of the concentration range of miRNA targets in clinical samples}

Fine-needle aspiration biopsy is a standard diagnostic procedure for tumor; th typically yields $10^{2}$ to $10^{4}$ cells in a sample. ${ }^{\mathrm{S}-\mathrm{S} 4}$ A single cell contains no more than $10^{3}$ copies of a miRNA of one type. ${ }^{\mathrm{S} 5, \mathrm{~S} 6}$ Thus, the total number of miRNA copies in a typical fine-needle biopsy sample is as few as $10^{5}-10^{7}$. A minimal volume of the lysis buffer required for complete lysis and for the following analysis is approximately $100 \mu \mathrm{L}$. As a result, we can estimate miRNA concentration in a fine-needle biopsy sample to be in a 0.01 to $1 \mathrm{pM}$ range, which is below the LOQ of the CEbased DQAMmiR. ${ }^{\mathrm{S} 7}$

\section{Note S2. Sequences of miRNA targets and their PNA probes}

The sequences of two miRNAs, miR-20b and miR-100, and their complementary PNA probes are presented in Table S1. The Alexa Fluor 647 dye is conjugated to the $\mathrm{N}$ terminus of the PNA strand via two O-linkers, which can improve the solubility of PNA probes in water.

Table S1. Sequences of target miRNAs and their fluorescently labeled complementary PNA hybridization probes

\begin{tabular}{|c|c|c|}
\hline Targets & Sequence of targets $\left(5^{\prime}-3^{\prime}\right)$ & Sequence of its complementary PNA probe (N'-C') \\
\hline miR-20b & $\begin{array}{l}\text { CAA-AGU-GCU-CAU-AGU- } \\
\text { GCA-GGU-AG }\end{array}$ & $\begin{array}{l}\text { AlexaFluor647-OO-CTA-CCT-GCA-CTA-TGA-GCA- } \\
\text { CTT-TG }\end{array}$ \\
\hline $\operatorname{miR}-100$ & $\begin{array}{l}\text { AAC-CCG-UAG-AUC-CGA- } \\
\text { ACU-UGU-G }\end{array}$ & $\begin{array}{l}\text { AlexaFluor647-OO-CAC-AAG-TTC-GGA-TCT-ACG- } \\
\text { GGT-T-Gly-Thr-Gly-Ala-Gly }\end{array}$ \\
\hline
\end{tabular}




\section{Note S3. Determination of the stop time, $t_{\mathrm{st}}$, for the FASS-ITP step in FASS-ITP-CE}

In FASS-ITP-CE, the preconcentration step should be stopped before the concentrated analytes reach the capillary end to prevent them from eluting from the capillary before the start of the separation step (Figure 2B). Our task was to find a way to stop FASS-ITP before the concentrated analytes leave the capillary with as little residual TE remaining as possible as it could deteriorate the quality of CE separation. After exploring a number of options, we focused on the value of electric current as an indicator of completion of the FASS-ITP step and that can be used to determine stop time, $t_{\mathrm{st}}$, as described elsewhere. ${ }^{\mathrm{S} 8}$ The displacement of both the low-conductivity sample matrix and TE by LE in the capillary during FASS-ITP is accompanied by a gradual increase in the electric current. There is a time-point on the current versus time dependence where the slope abruptly changes from finite to zero (Figure S1). This time is thought to correspond to the exact moment of the completion of electrolyte displacement. Thus, we could stop FASS-ITP at this time and resume the CE separation subsequently.

As shown in Figure $\mathbf{S 1}, t_{\mathrm{st}}$ was determined to be $5.00 \mathrm{~min}$ for analyzing samples in the pure buffer (left panel). This time increased to $5.25 \mathrm{~min}$ for the analysis of samples in the cell lysate because the effective mobility of the electroosmotic flow (EOF) during the step of FASS-ITP decreased for the cell lysate due to the presence of extra salts in the sample plug from the cellular contents (Figure S1, right panel). The lower EOF mobility results in a longer time for electrolyte displacement to complete. Considering the variability of biological matrices in clinical samples, we, thus, suggest that $t_{\mathrm{st}}$ should be determined specifically for each sample via an extra FASS-ITP run prior to FASS-ITP-CE analysis as described here.
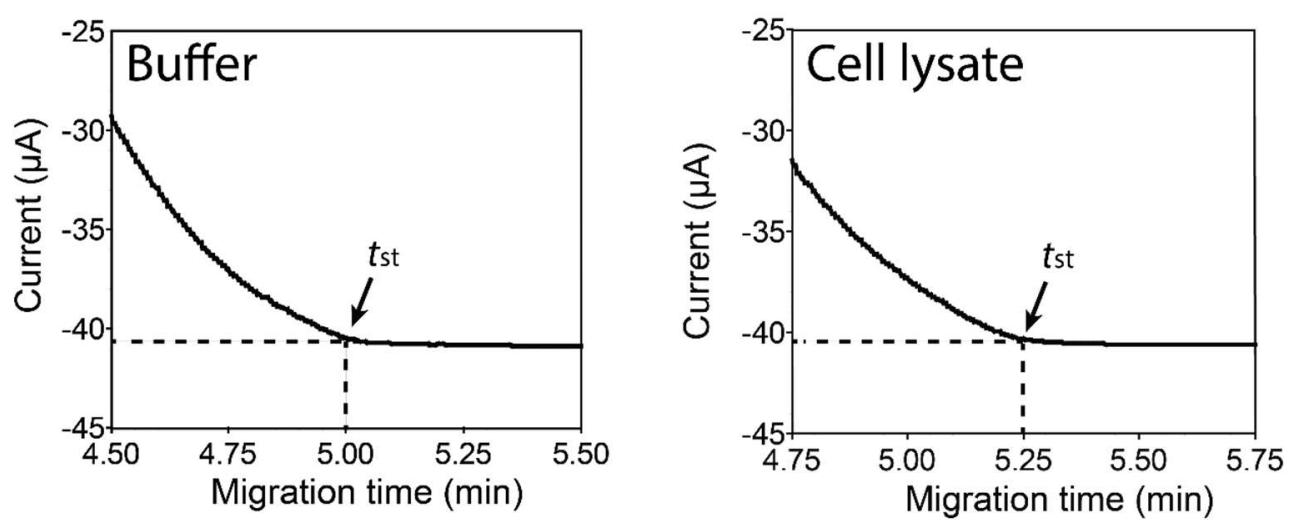

Figure S1. Determination of the time-point $\left(t_{\mathrm{st}}\right)$ for stopping in preconcentration by FASS-ITP and staring CE by applying an electric field with a reversed polarity. Left panel: samples in pure buffer. Right panel: cell lysate samples. 


\section{Note S4. Relative quantum yields measurements}

Two aspects must be considered to facilitate accurate quantitation of target miRNAs: (i) the potential difference in the quantum yield between the PNA probes and (ii) the potential influence of miRNA binding to the PNA probe on the quantum yield of the due on the probe. Thus, relative quantum yield $\left(q_{\mathrm{H}}\right)$ of each PNA-miRNA hybrid with respect to its unbound PNA probe was determined as explained in our previous work (Table S2). ${ }^{\mathrm{S} 7}$ The fluorescence intensity of each PNA probe was also normalized by determining its quantum yield $\left(q_{\mathrm{P}}\right)$ with respect to the PNA probe complementary to miR-100, which was used as a reference (Table S3). ${ }^{\text {S9 }}$

Table S2. Relative quantum yields of PNA-miRNA hybrids $\left(q_{\mathrm{H}}\right)$ with respect to their unbound PNA probes. Standard deviations from mean values were obtained from three experiments.

\begin{tabular}{|l|l|l|}
\hline PNA-miRNA hybrid & PNA-miR-100 & PNA-miR-20b \\
\hline$q_{\mathrm{H}}$ & $0.28 \pm 0.03$ & $0.26 \pm 0.01$ \\
\hline
\end{tabular}

Table S3. Relative quantum yields of the PNA probes $\left(q_{\mathrm{P}}\right)$ to the PNA probe corresponding to miR-100 for signal normalization. Standard deviations from mean values were obtained from three experiments.

\begin{tabular}{|c|c|c|}
\hline PNA Probe & PNA probe of miR-100 & PNA probe of miR-20b \\
\hline$q_{\mathrm{P}}$ & 1 & $0.88 \pm 0.02$ \\
\hline
\end{tabular}

\section{Note S5. ITP-CE for DQAMmiR}

The capillary was prefilled with the TE buffer with pressure of $20 \mathrm{psi}(137.9 \mathrm{kPa})$ for $1 \mathrm{~min}$. Sample in the LE buffer (100 mM Tris-Cl, 20\% (v/v) ACN, pH 8.2) was injected from the capillary outlet by a pressure pulse of 3 psi for $99 \mathrm{~s}$. The buffer in the outlet was switched to a clean LE buffer and an electric field of $375 \mathrm{~V} / \mathrm{cm}$ was applied with positive polarity at the capillary outlet. The voltage was turned off at the time point when the displacement of TE with LE was completed, which can be determined by observing the current profile of the run. ${ }^{\mathrm{S} 8}$ Buffer in the inlet was switched to LE buffer and an electric field of $375 \mathrm{~V} / \mathrm{cm}$ was applied with positive polarity at the capillary inlet. The unreacted PNA probes and the PNA-miRNA hybrids were then separated and detected by CE-LIF as in the standard CE-based DQAMmiR. 


\section{Note S6. Purification of the PNA probes}

The presence of fluorescent impurities in the PNA probes significantly increases the LOQ for miRNA detection in FASS-ITP-CE. As evident from Figure S2, bottom trace, the PNA probes from the supplier contained a significant amount of impurities overlapping with the hybrids in the electropherograms. This would influence the accuracy of the peak areas of the hybrids while target concentrations are low, and consequently, lead to errors in quantitation and poor (high) LOQ. Ideally, the impurities in the PNA probe stocks can be removed by collecting fractions at the CE outlet as they are separable from the PNA probes in CE. However, because only a small volume of sample can be injected in CE for separation, the probes in the collected fractions would be diluted by multiple orders of magnitude, which makes it difficult to be used PNA purified in such a way in the following FASS-ITP-CE experiments.

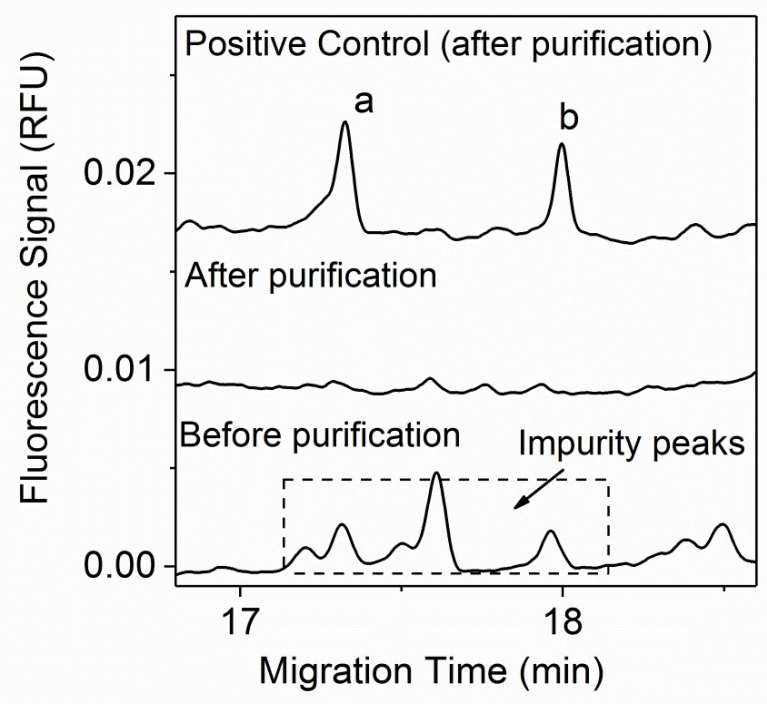

Figure S2. Electropherograms of the negative control samples before purification (bottom trace) and after purification (middle trace). The negative control samples were composed of $5 \mathrm{nM}$ PNA-20b and $1 \mathrm{nM}$ PNA-100 only without miRNA targets. Samples composed of 1 pM miR-20b, 1 pM miR-100, 5 nM PNA$20 \mathrm{~b}$ and $1 \mathrm{nM}$ PNA-100 was also analyzed as positive control (top trace). The purified PNA probes were used in this positive control sample. Peak assignment: a) PNA-miR-100 hybrid; b) PNA-miR-20b hybrid.

To address this issue, we developed a protocol to remove the impurities in the PNA probe stocks based not on CE but on our FASS-ITP-CE procedure. In FASS-ITP-CE, the injected sample volume is 120 times greater than that in a standard CE run. PNA probes in a large sample plug are concentrated into a narrow zone by FASS while the impurities are being focused by the hyphenated 
process of FASS-ITP. Thus, the PNA probes can be separated from the impurities in the subsequent $\mathrm{CE}$. Thus, we could remove the impurities by collecting the fraction of the concentrated PNA probes at the capillary outlet during CE separation. As a 120-time volume was injected, the PNA probes in the collected fraction in this method would supposedly be approximately 120 times less diluted than that in normal CE. Furthermore, we can increase the concentration of the purified PNA probes by collecting the fractions of the PNA probes from multiple runs into a single tube. For example, by collecting the fractions from 5 repeated runs, we successfully obtained a solution of purified PNA probe for miR-100 with a concentration of $1.2 \mu \mathrm{M}$ from a stock with a concentration of $6 \mu \mathrm{M}$. The high-concentration purified probes could then be quantified by UVabsorbance and stored for their use in the following analysis. In Error! Reference source not found., we also show the result of an experiment in which we analyzed a sample containing purified PNA probes only (middle trace). It was clear that the impurity levels in the PNA probes of the same concentrations were significantly lower than those before purification. We also analyzed a sample containing each miRNA target at a concentration of $1 \mathrm{pM}$ by using the purified PNA probes. The result (Figure S2, top trace) demonstrated that the miRNA targets of 1 pM could be accurately quantitated by using the purified PNA probes. This result clearly indicated that lowering the level of impurities via purification of PNA probes provided by a supplier would allow us to quantitate hybrids peaks with concentrations below $1 \mathrm{pM}$ accurately. 


\section{Note S7. Electropherograms and results of miRNA quantification by FASS-ITP-CE}

Figure S3 shows the electropherograms for FASS-ITP-CE measurements of samples with a varying miRNA concentration. The quantification results of these measurements are shown in Table S4.
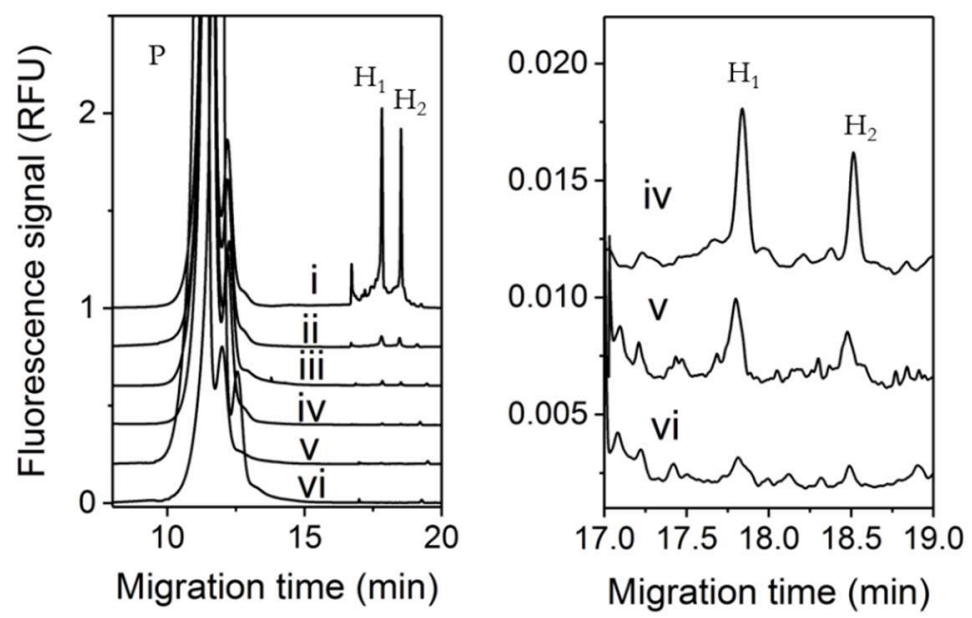

Figure S3. Electropherograms for FASS-ITP-CE of samples in pure buffer. P stands for excess PNA probes; $\mathrm{H}_{1}$ stands for PNA-miR-100 hybrid; and $\mathrm{H}_{2}$ stands for PNA-miR-20b hybrid. Electropherograms i-vi (left panel) correspond to target concentrations of 100, 10, 5, 1, 0.5, and $0.1 \mathrm{pM}$, respectively. Electropherograms for targets concentrations of $1,0.5$, and $0.1 \mathrm{pM}$ are zoomed-in (right panel) for clarity.

Table S4. Measured concentrations of two miRNAs (miR-100 and miR-20b) shown with respect to their actual concentration as determined by light absorbance at $260 \mathrm{~nm}$; standard deviations from mean values were obtained from three independent experiments

\begin{tabular}{ccc}
\hline \multirow{2}{*}{$\begin{array}{c}\text { Actual miRNA } \\
\text { Concentration }(\mathrm{pM})\end{array}$} & \multicolumn{2}{c}{$\begin{array}{c}\text { Measured miRNA Concentration } \\
(\mathrm{pM}, \text { Mean } \pm \text { Standard Deviation })\end{array}$} \\
\cline { 2 - 3 } & miR-20b & miR-100 \\
\hline 100 & $92.1 \pm 0.7$ & $107.0 \pm 0.6$ \\
10 & $9.9 \pm 0.8$ & $9.7 \pm 0.5$ \\
5 & $4.7 \pm 0.1$ & $5.1 \pm 0.1$ \\
1 & $1.1 \pm 0.1$ & $0.9 \pm 0.1$ \\
0.5 & $0.52 \pm 0.02$ & $0.50 \pm 0.01$ \\
0.1 & $0.11 \pm 0.01$ & $0.11 \pm 0.01$ \\
\hline
\end{tabular}




\section{Note S8. Protocol of cell culture}

RWPE- 1 and $22 \mathrm{Rv} 1$ cells were grown in an incubator at $37{ }^{\circ} \mathrm{C}$ in the atmosphere of $5 \% \mathrm{CO}_{2}$. The RWPE-1 cells were grown in Keratinocyte-Serum Free Media (K-SFM) with L-glutamine purchased from ThermoFisher Scientific (Burlington, ON, Canada) supplemented with human recombinant epidermal growth factor and bovine pituitary extract which were supplied with the K-SFM. The 22Rv1 cells were grown in Roswell Park Memorial Institute (RPMI) 1640 Medium purchased from ThermoFisher Scientific (Burlington, ON, Canada) with a supplement of $100 \mathrm{IU} \cdot \mathrm{mL}^{-1}$ penicillin, $100 \mu \mathrm{g} \cdot \mathrm{mL}^{-1}$ streptomycin, and $10 \%$ fetal bovine serum purchased from Invitrogen (Burlington, Ontario, Canada). When cells covered roughly $80 \%$ of the plate they were washed with PBS, trypsinized to detach them from the bottom of the dish and centrifuged at $150 \times \mathrm{g}$ for $5 \mathrm{~min}$. The cells were washed twice with PBS and counted using a hemocytometer. The cells were aliquoted into 100,000 cells per pellet and stored at $-80^{\circ} \mathrm{C}$.

\section{Note S9. Analyzing miRNAs in crude cell lysates}

Each cell pellet containing 10,000 cells was lysed by adding a $100-\mu \mathrm{L}$ volume of lysis solution composed of $0.1 \%(\mathrm{v} / \mathrm{v})$ Triton X-100, $5 \mathrm{nM}$ PNA-20b, $1 \mathrm{nM}$ PNA-100 in the buffer of $1 \mathrm{mM}$ Tris-Cl, 20\% (v/v) ACN, $\mathrm{pH} 8.2$, giving a final concentration of the cell contents in the lysate equivalent to 100,000 cells per $1 \mathrm{~mL}$. The lysate was then mixed with the probes, and the mixture was incubated for hybridization and analyzed by FASS-ITP-CE according to the Materials and Methods in the main text. The quantification results for measuring miR-100 and miR-20b are shown in Table S5. To account for a potential effect of the cell contents on miRNA quantification, we also spiked $0.5 \mathrm{pM}$ of each miRNA target into the $22 \mathrm{Rv} 1$ cell lysate. The FASS-ITP-CE measurement was performed as previously explained (Figure S4). The detection of the spiked-in miRNA targets validated the detection of miRNA from the cell lysate and allowed us to conclude that the effect of the cell lysate contents on miRNA quantitation was negligible. 
Table S5. Concentrations of two miRNAs (miR-100 and miR-20b) in RWPE-1 cell lysates and 22Rv1 cell lysates determined by FASS-ITP-CE.

\begin{tabular}{|c|c|c|c|c|c|c|}
\hline & & $\begin{array}{c}\text { Rep1 } \\
(\mathrm{pM})\end{array}$ & $\begin{array}{c}\text { Rep2 } \\
(\mathrm{pM})\end{array}$ & $\begin{array}{c}\text { Rep3 } \\
(\mathrm{pM})\end{array}$ & $\begin{array}{c}\text { Mean } \\
(\mathrm{pM})\end{array}$ & $\begin{array}{c}\text { Standard } \\
\text { Deviation } \\
(\mathrm{pM})\end{array}$ \\
\hline \multirow{2}{*}{$\begin{array}{c}\text { RWPE-1 } \\
\text { Cell lysate }\end{array}$} & {$[\mathrm{miR}-100]$} & $0.08^{*}$ & $0.08^{*}$ & $0.09^{*}$ & 0.08 & 0.01 \\
\cline { 2 - 7 } & {$[\mathrm{miR}-20 \mathrm{~b}]$} & $\begin{array}{c}\text { Below } \\
\text { LOD }\end{array}$ & $\begin{array}{c}\text { Below } \\
\text { LOD }\end{array}$ & $\begin{array}{c}\text { Below } \\
\text { LOD }\end{array}$ & $\begin{array}{c}\text { Not } \\
\text { applicable }\end{array}$ & $\begin{array}{c}\text { Not } \\
\text { applicable }\end{array}$ \\
\hline $\begin{array}{c}\text { 22Rv1 } \\
\text { Cell lysate }\end{array}$ & {$[\mathrm{miR}-100]$} & 0.12 & 0.12 & 0.14 & 0.13 & 0.01 \\
\cline { 2 - 7 } & {$[\mathrm{miR}-20 \mathrm{~b}]$} & $0.09^{*}$ & 0.10 & 0.11 & 0.10 & 0.01 \\
\hline
\end{tabular}

*Below LOQ but higher than LOD.

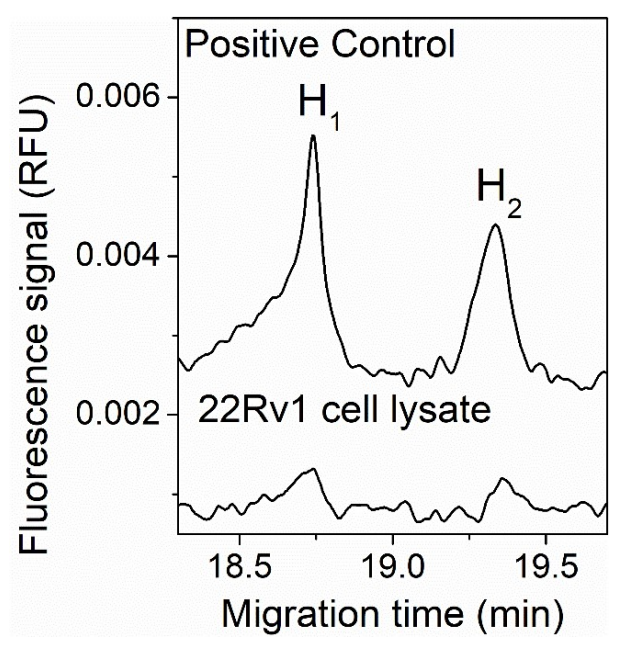

Figure S4. Electropherograms for FASS-ITP-CE measurements of miR-100 and miR-20b in the 22Rv1 cell lysate (bottom trace) and a positive control sample (top trace). $\mathrm{H}_{1}$ stands for the PNA-miR-100 hybrid and $\mathrm{H}_{2}$ stands for the PNA-miR-20b hybrid. The positive control sample was prepared by spiking $50 \mathrm{pM}$ miR$20 \mathrm{~b}$ and miR-100 into the $22 \mathrm{Rv} 1$ cell lysate. 


\section{Supporting References}

(S1) Stomper, P. C.; Nava, M. E.; Budnick, R. M.; Stewart, C. C. Specimen MammographyGuided Fine-Needle Aspirates of Clinically Occult Benign and Malignant Lesions. Analysis of cell number and type. Investig. Radiol. 1997, 32, 277-281.

(S2) Wiedswang, G.; Borgen, E.; Karesen, R.; Naume, B. Detection of Isolated Tumor Cells in BM from Breast-Cancer Patients: Significance of Anterior and Posterior Iliac Crest Aspirations and the Number of Mononuclear Cells Analyzed. Cytotherapy 2003, 5, 40-45.

(S3) M.A. Lewandowska, W. Jozwicki, C. Jochymski, J. Kowalewski, Application of PCR Methods to Evaluate EGFR, KRAS and BRAF Mutations in a Small Number of Tumor Cells in Cytological Material from Lung Cancer Patients, Oncol. Rep. 2013, 30, 1045-1052.

(S4) Mascalchi, M.; Falchini, M.; Maddau, C.; Salvianti, F.; Nistri, M.; Bertelli, E.; Sali, L.; Zuccherelli, S.; Vella, A.; Matucci, M.; Voltolini, L.; Pegna, A. L.; Luconi, M.; Pinzani, P.; Pazzagli, M. Prevalence and Number of Circulating Tumour Cells and Microemboli at Diagnosis of Advanced NSCLC. J. Cancer Res. Clin. Oncol. 2016, 142, 195-200.

(S5) Liang, Y.; Ridzon, D.; Wong, L.; Chen, C. Characterization of microRNA Expression Profiles in Normal Human Tissues. BMC Genom. 2007, 8, 166.

(S6) Lu, J.; Tsourkas, A. Imaging Individual microRNAs in Single Mammalian Cells in Situ. Nucleic Acids Res. 2009, 37, e100.

(S7) Hu, L.; Anand, M.; Krylova, S. M.; Yang, B. B.; Liu, S. K.; Yousef, G. M.; Krylov, S. N. Direct Quantitative Analysis of Multiple microRNAs (DQAMmiR) with Peptide Nucleic Acid Hybridization Probes. Anal. Chem. 2018, 90, 14610-14615.

(S8) Wegman, D. W.; Ghasemi, F.; Khorshidi, A.; Yang, B. B.; Liu, S. K.; Yousef, G. M.; Krylov, S. N. Highly-Sensitive Amplification-Free Analysis of Multiple miRNAs by Capillary Electrophoresis. Anal. Chem. 2015, 87, 1404-1410. 